Case report

\title{
Tocilizumab in MOG-antibody spectrum disorder: a case report
}

\author{
G. Novi ${ }^{\mathrm{a}, \mathrm{e}}$, M. Gastaldi ${ }^{\mathrm{b}}$, D. Franciotta ${ }^{\mathrm{b}}, \mathrm{G}$. Pesce $^{\mathrm{c}, \mathrm{e}}$, L. Benedetti $^{\mathrm{a}, \mathrm{e}, 1}$, A. Uccelli ${ }^{\mathrm{d}, \mathrm{e}, *, 1}$ \\ ${ }^{a}$ Department of Neurosciences, Rehabilitation, Ophthalmology, Genetics, Maternal and Child Health Unit, University of Genoa, Genoa, Italy \\ ${ }^{\mathrm{b}}$ Neuroimmunology Laboratory, IRCCS Mondino Foundation, Pavia, Italy \\ ${ }^{\mathrm{c}}$ Autoimmunity Laboratory DiMI, University of Genoa, Genoa, Italy \\ ${ }^{\mathrm{d}}$ Department of Neurosciences, Rehabilitation, Ophthalmology, Genetics, Maternal and Child Health Unit, Center of Excellence for Biomedical Research, University of \\ Genoa, Genoa, Italy \\ ' Ospedale Policlinico San Martino-IRCCS, Genoa, Italy
}

\section{A R T I C L E I N F O}

\section{Keywords:}

Tocilizumab

MOG

Neuromyelitis optica spectrum disorders

\begin{abstract}
A B S T R A C T
Background: Myelin oligodendrocyte glycoprotein antibody-related spectrum disorders (MOG-SD) are a heterogeneous group of inflammatory demyelinating diseases of the central nervous system, usually responsive to conventional immunosuppressive therapies. However, knowledge about treatment of non-responder patients is scarce.

Methods: We report on a 20-year-old MOG-SD patient who experienced clinical deterioration despite rituximabinduced B-cell depletion.

Results: Rescue therapy with tocilizumab (TCZ) prevented further relapses, with reduction of spinal-cord load on MRI, and a remarkable reduction of disability at the two-year follow-up.

Conclusion: Our observations suggest that TCZ could induce clinico-radiologic improvements, which make it as an option for the treatment of MOG-SD.
\end{abstract}

Myelin oligodendrocyte glycoprotein (MOG) antibody-related spectrum disorders (MOG-SD) are a phenotypically heterogeneous group of diseases that include optic neuritis, myelitis, brainstem encephalitis, and acute disseminated encephalomyelitis (ADEM)-like presentations. MOG-SD can be considered a disease entity distinct from multiple sclerosis (MS) and aquaporin-4 (AQP4)-antibody-positive neuromyelitis optica spectrum disorders ${ }^{1}$.

In analogy with AQP4 antibody-positive NMOSD, therapies approved for MS can be ineffective or even harmful in MOG-SD (Jarius et al., 2018). Patients with NMOSD refractory to first-line conventional drugs (e.g., rituximab and azathioprine) can benefit from treatments with the interleukin-6 receptor (IL-6r) blocking monoclonal antibody tocilizumab (TCZ) (Trebst et al., 2014). Three studies showed the potential efficacy of TCZ in small groups of highly relapsing, and mainly AQP4 antibody-positive NMOSD patients who were unresponsive to conventional therapies (Ayzenberg et al., 2013; Araki et al., 2014; Ringelstein et al., 2015).

We report on the first patient with highly active and rituximabunresponsive MOG-SD who was treated with TCZ.
In August 2015, a 20-year-old male without any relevant medical history, developed right eye visual impairment that rapidly involved the fellow eye. Neurological examination showed only visual disturbances, with a visual acuity of 20/25 in both eyes. Brain MRI disclosed unremarkable results and a bilateral optic neuritis was diagnosed. The patient was treated with oral prednisone $(50 \mathrm{mg} /$ day halved every 10 days) for 1 month, with normalization of visual acuity. In October 2015, the patient came to our attention after developing lower limbs sensory deficits with urinary retention. Neurological examination showed a left side hemiparesis with tetra-hyperreflexia, upper limbs hypopallesthesia, and abdominal hyperesthesia (T8 sensory level). Spinal MRI showed non-enhancing, longitudinal, and thin T2 hyperintense lesions involving T4-T6, T8-T10, and T12-L1 segments (Fig. 1A) with lumbar leptomeningeal Gadolinium (Gd) enhancement. CSF analysis disclosed normal cell count and total protein concentration, and identical oligoclonal IgG bands in serum and CSF (mirror pattern). Infectious myelitis was excluded on the ground of serologic and virologic CSF results. Serum anti-nuclear antibodies, anti-extractable nuclear antigens, and AQP4 antibodies tested negative, whereas MOG

\footnotetext{
*Corresponding author at: Department of Neurology, Rehabilitation, Ophthalmology, Genetics, Maternal and Child Health (DINOGMI), University of Genoa, Clinica neurologica, Largo Paolo Daneo3, Genoa 16132, Italy.

E-mail addresses: giovanninovi@gmail.com (G. Novi), matteo.gastaldi@mondino.it (M. Gastaldi), diego.franciotta@mondino.it (D. Franciotta), pescegpl@unige.it (G. Pesce), luanabenedetti@libero.it (L. Benedetti), auccelli@neurologia.unige.it (A. Uccelli).

${ }^{1}$ These senior authors equally contributed to the manuscript.
} 


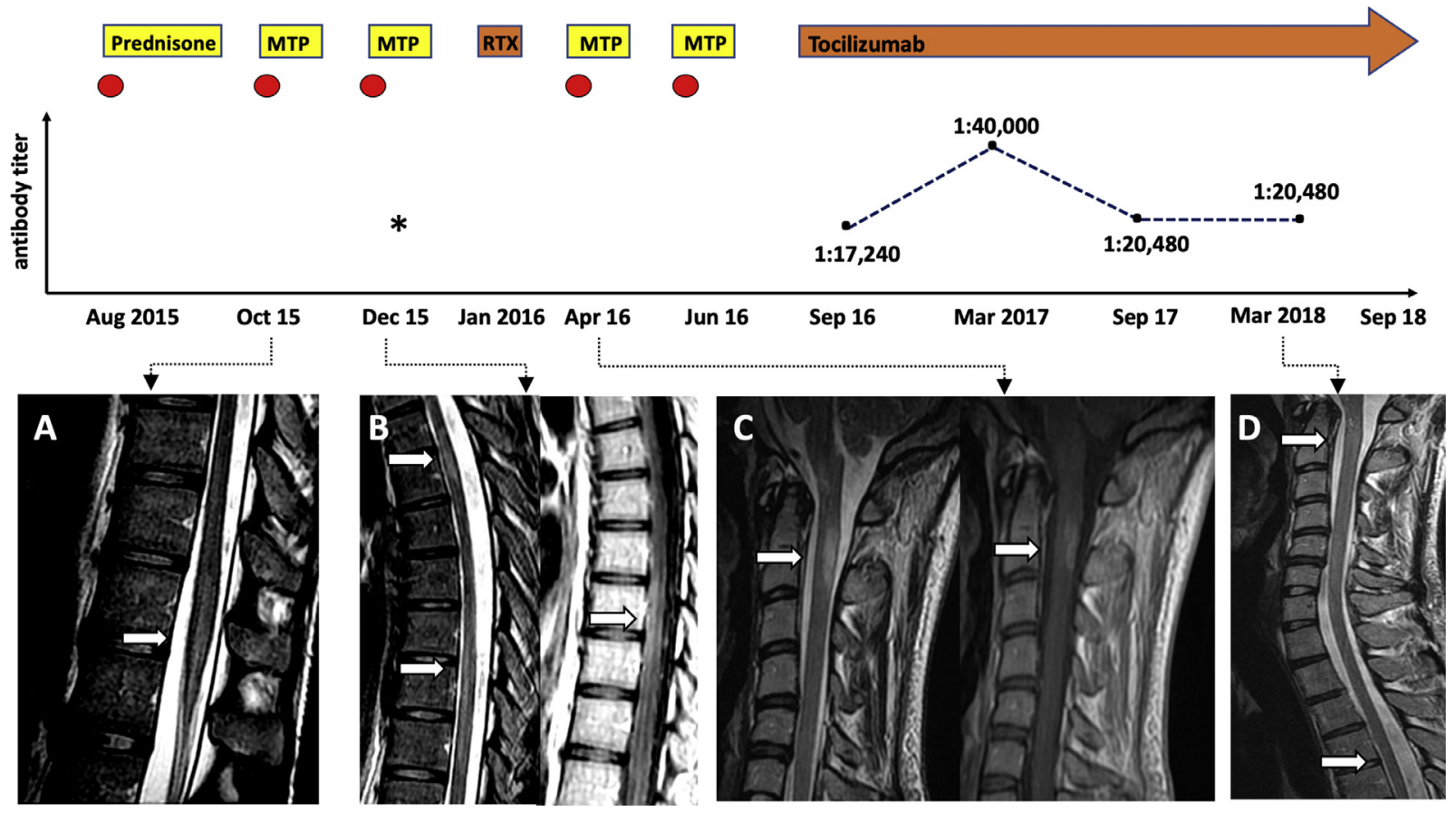

Fig. 1. Disease course, treatments, MOG antibody titers, and spinal MRI scans.

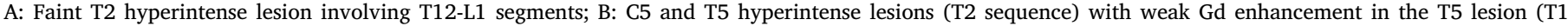

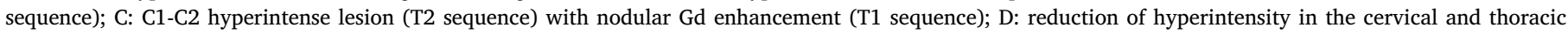

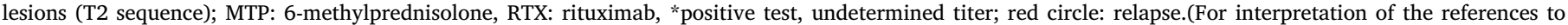
colour in this figure legend, the reader is referred to the web version of this article.)

antibodies were positive for both total IgG and IgG1 (in-house cellbased assays, using HEK293T cells transfected with human full-length MOG). A diagnosis of MOG-SD was made and the patient was treated with high-dose intravenous steroids ( $1 \mathrm{~g}$ 6-methylprednisolone/day for 5 days), with partial improvement of symptoms. In December 2015, a sensory relapse occurred. Spinal MRI showed two new Gd enhancing lesions involving C5 and T5 segments (Fig. 1B). High-dose intravenous steroids were administered, with symptoms resolution. The patient then underwent two rituximab cycles ( $1 \mathrm{~g}$ /infusion, 15 days apart) in January 2016. Despite a complete B-cell depletion, two relapses occurred, manifesting as cervical myelitis (Fig. 1C), and as right eye optic neuritis, respectively at 3 and 5 months after rituximab induction. Therefore, in September 2016, treatment with TCZ ( $8 \mathrm{mg} / \mathrm{kg}$ every 4 weeks) was initiated. At 24-month follow-up, no new relapses had occurred, and the patient was substantially asymptomatic (only lower limbs hyperreflexia at neurological examination). Spinal MRI showed a reduction of cervical and thoracic lesions (Fig. 1D). Serial serum MOG antibody determinations, performed every 6 months, showed the persistence of high antibody titers (Fig. 1; median value: 1:18,740; range: 1:17,280-1:40,000). No adverse events occurred throughout the TCZ therapy, still ongoing (Ringelstein et al., 2015).

This case suggests that TCZ can be an effective therapeutic option in rituximab refractory MOG-SD. The mechanisms of action underlying the effects of TCZ in our MOG-SD patients are not known but may suggest different pathogenic mechanisms between MOG-SD and NMOSD. Both AQP4 and MOG antibody-associated disorders seem to share similar cytokine signatures, characterized by higher CSF IL-6 concentrations $v$ s. MS patients and healthy controls (Kaneko et al., 2018). IL-6 has a crucial role in promoting the survival of circulating plasmablasts, a major source of AQP4 antibodies in NMOSD (Chihara et al., 2011). In addition, IL-6 levels are increased in serum and CSF samples during NMOSD relapses (Uzawa et al., 2010), and responses to TCZ in rituximab unresponsive AQP4 antibody-positive NMOSD have been reported (Ayzenberg et al., 2013; Araki et al., 2014; Ringelstein et al., 2015).
The persistence of high MOG antibody titers in our patient despite the clinico-radiologic improvements suggest that they could be a biomarker of disease, but not of response to therapy, and further stimulates the debate about whether human MOG-antibodies are pathogenic or epiphenomenic. A further clue favoring the epiphenomenic hypothesis comes from the post-TCZ reduction of spinal cord lesions observed in our patient, in contrast with what suggested by the complementmediated pathogenic effects of a subset of MOG antibodies in an animal model (Marignier et al., 2017), and with what observed in AQP4 antibody-positive NMOSD (Marignier et al., 2017).

Further studies on TCZ-treated MOG-SD patients, with and without preceding rituximab cycles, should be performed to confirm our observations. If confirmed by other studies, TCZ should be considered as a possible option to treat NMOSD individuals regardless of the phenotypic presentations.

\section{Conflict of interest}

Antonio Uccelli received grants and contracts from Fondazione Italiana Sclerosi Multipla (FISM), Novartis, Fondazione Cariplo, Italian Ministry of Health; received honoraria or consultation fees from Biogen, Roche, Teva, Merck-Serono, Genzyme, Novartis.

\section{Funding}

This manuscript received no funding.

The manuscript received approval from local ethic committee.

\section{References}

Jarius, S, Paul, F, Aktas, O, et al., 2018. MOG encephalomyelitis: international recommendations on diagnosis and antibody testing. J. Neuroinflammation 15 (May), 134. https://doi.org/10.1186/s12974-018-1144-2.

Trebst, C, Jarius, S, Berthele, A, et al., 2014. Update on the diagnosis and treatment of neuromyelitis optica: recommendations of the Neuromyelitis Optica Study Group (NEMOS). J. Neurol. 261, 1-16. https://doi.org/10.1007/s00415-013-7169-7. 
Ayzenberg, I, Kleiter, I, Schroder, A, et al., 2013. Interleukin 6 receptor blockade in patients with neuromyelitis optica nonresponsive to anti-CD20 therapy. JAMA Neurol. 70 (January), 394-397. https://doi.org/10.1001/jamaneurol.2013.1246.

Araki, M, Matsuoka, T, Miyamoto, K, et al., 2014. Efficacy of the anti-IL-6 receptor antibody tocilizumab in neuromyelitis optica: a pilot study. Neurology 82 (March), 1302-1306. https://doi.org/10.1212/WNL.0000000000000317.

Ringelstein, M, Ayzenberg, I, Harmel, J, et al., 2015. Long-term therapy with interleukin 6 receptor blockade in highly active neuromyelitis optica spectrum disorder. JAMA Neurol. 72 (May), 756-763. https://doi.org/10.1001/jamaneurol.2015.0533.

Kaneko, K, Sato, DK, Nakashima, I, et al., 2018. CSF cytokine profile in MOG-IgG + neurological disease is similar to AQP4-IgG + NMOSD but distinct from MS: a crosssectional study and potential therapeutic implications. J. Neurol. Neurosurg.
Psychiatry 89 (June), 927-936. https://doi.org/10.1136/jnnp-2018-317969.

Chihara, N, Aranami, T, Sato, W, et al., 2011. Interleukin 6 signaling promotes antiaquaporin 4 autoantibody production from plasmablasts in neuromyelitis optica. Proc. Natl. Acad. Sci. U S A 108 (February), 3701-3706. https://doi.org/10.1073/ pnas.1017385108.

Uzawa, A, Mori, M, Arai, K, et al., 2010. Cytokine and chemokine profiles in neuromyelitis optica: significance of interleukin-6. Mult. Scler. 16 (August), 1443-1452. https://doi.org/10.1177/1352458510379247.

Marignier, R, Cobo Calvo, A, Vukusic, S., 2017. Neuromyelitis optica and neuromyelitis optica spectrum disorders. Curr. Opin. Neurol. 30 (March), 208-215. https://doi.org/ 10.1097/WCO.0000000000000455 